\title{
Die Kopula werden und die Situationstypen ${ }^{1}$
}

\author{
Renate Steinitz \\ ZAS Berlin \\ Steinitz@zas.gwz-berlin.de
}

\begin{abstract}
The copula sein "be" in German, together with its complements, refers to a stative situation. Besides offering argument positions in its Semantic Form SF, it has no other function. Stative verbs are not specified with respect to the beginning or the end of a described situation or with respect to the state before or after. I will take the verb werden "become, get" to be a copular verb as well. The only difference to sein is that werden refers to a nonstative or changing situation. I argue that werden is underspecified in two respects. Like motion verbs and successive patient verbs (SUK verbs in Krifka (1989)) werden switches between an unlimited and a limited process (accomplishment) dependent on its complement (cf. älter werden "get older" / vorwärts gehen "go forward" / Tee trinken "drink tea" vs. alt werden "get old" / in das Zimmer gehen "go into the room"/ eine Tasse Tee trinken "drink a cup of tea"). But werden is even more underspecified than these verbs; it is the only verb which covers all nonstative situations, not only processes and accomplishments but also punctual transitions (achievements), cf. schwanger werden "get pregnant". Werden is anything but stative. Whether there is a target state implied or not, or whether the transition to this target state is extensible or atomic, is the result of the composition of the meaning of werden and its internal argument added by special meaning postulates. Hierarchically marked subtypes of situational arguments result as a side effect.
\end{abstract}

Wir sind alle Werdende alte Volksweisheit

\section{Kopula-Verben}

Neben anderen Argumenten ist in einem Vollverb ein Situationsargument s verankert, das für die temporale und aspektuelle Einordnung zuständig ist. Auch die Kopula verhält sich in dieser Hinsicht wie ein Vollverb ${ }^{2}$, aber damit ist im Wesentlichen ihre Aufgabe auch erschöpft. Ihre Eigenbedeutung beschränkt sich auf klassenbildende Bedeutungskomponenten, die z.B. Zustandsverben und Inchoativa unterscheiden. Vollverben haben dagegen außerdem noch klassenbelegende Bedeutungskomponenten.

In der Bedeutung der Kopula ist wie bei transitiven Verben und Bewegungs- und Positionsverben eine Variable für ein syntaktisches Komplement verankert.

- Bei transitiven Verben ist es ein Individuen-Argument, im Lexikoneintrag eines Verbs als Individuenvariable $\mathrm{x}$ verankert und durch eine referierende DP zu realisieren.

- Bei lokativen und Kopulaverben ist es ein Prädikat-Argument, als Prädikatenvariable P repräsentiert und durch eine nicht selbständig referierende Phrase realisierbar, vgl. Steinitz

\footnotetext{
' Ich habe vielen Kollegen in meinem Umkreis zu danken, und sei es dafür, daß sie mich geduldig von meinen Abwegen abzubringen suchten. Logischerweise danke ich besonders denen, die wie G. Jäger, E. Lang, A. Steube und I. Zimmermann mich getreulich und kritisch begleitetet haben. Den anonymen Gutachtern ist es zu verdanken, daß ich einige Fehler ausgemerzt und den Aufsatz radikal gekürzt habe.

${ }^{2}$ Es ist strittig, ob jedem Verb ein Situationsargument zukommt oder ob Statives wie ähnlen, heißen und eben auch sein kein solches Argument haben. Diese Problematik lasse ich beiseite.
} 
c) schwanger werden : in Berlin ankommen

und den diesen Verbkonstruktionen zugeordneten (in Abschnitt 4 erläuterten) Situationstypen

a) Accomplishment, b) Prozeß und c) Achievement, ist die Frage berechtigt,

- ob die telische Bedeutung von BECOME in allen drei Kontexten zutreffend ist und

- ob die übliche Abbildung eines telischen Prädikats auf die unterschiedlichen Situationstypen den Fakten gerecht wird.

\section{Die Bedeutung von werden nach Auffassung A:}

Nach gängiger Auffassung (fortan Auffassung A) ist die Semantische Form (SF) von werden vom semantisch unmarkierten sein durch das klassenbildende Prädikat BECOME unterschieden.

lwerden/ $\quad \lambda \mathrm{P} \lambda \mathrm{x} \lambda \mathrm{s}[\mathrm{s}$ INST $[\mathrm{BECOME}[\mathrm{P} \mathrm{x}]]]$

BECOME ist ein telisches Prädikat, dessen Bedeutung z.B. in von Stechow (1996:96, (3-11)) qua Wahrheitsbedingung definiert werden kann

$\|\mathrm{BECOME}\|(\mathrm{P})(\mathrm{e})=1$, iff $\mathrm{e}$ is the smallest event such that $\mathrm{P}$ is not true of the pre-state of e but $\mathrm{P}$ is true of the target state of $\mathrm{e}$.

Diese Definition entspricht genau den in der Generativen Semantik der siebziger Jahre üblichen Termen von durch BECOME impliziertem Nachzustand $\mathrm{P}$ und damit vorausgesetztem komplementären Vorzustand Neg P und gilt für telische Prädikate wie altern, sterben, ankommen, alt/groß werden, schwanger werden. Der Baustein BECOME soll aber auch in Prozeßprädikaten wie wachsen, größer werden in (2b) enthalten sein.

\section{Mein Gegenentwurf, fortan Auffassung B:}

Der Stein des Anstoßes ist die Zuständigkeit des telischen Prädikats BECOME auch für die Prozeßverben wachsen, größer werden.

Werden ist nach meiner Auffassung vom statischen Prädikat sein nicht durch Telizität (Zustandswechsel) unterschieden, sondern dadurch, daß es ein unspezifisches nicht-statisches oder „Veränderungsprädikat“ ist. Es ändert sich etwas an der Eigenschaft oder am Zustand eines Individuums $\mathrm{x}$, doch werden bestimmt nicht,

- ob die Veränderung zu einem anderen Zustand führt oder nicht, und falls ja,

- ob dieser Übergang punktuell ist oder nicht.

Festlegungen dieser Art kommen durch die jeweilige Spezifikation des Prädikat-Arguments P in die Gesamtbedeutung von werden-Konstruktionen, das will ich im Fortgang der Argumentation deutlich machen.

Die Bedeutung von werden ist also bezüglich Telizität unbestimmt. Nur um die mit BECOME eingeschliffene Assoziation "Telizität" zu vermeiden, benutze ich statt dessen das semantische Prädikat CHANGE als Bedeutungskomponente von werden. Das ist keine terminologische Spielerei, CHANGE Px ist die Basis- oder invariante Bedeutungskomponente von werden in allen adjektivischen (und anderen) werden-Konstruktionen.Sie ist Teil sowohl von Ausdrücken, die eine Situation als einen unbegrenzten Prozeß denotieren (größer werden), als auch von solchen, die eine Begrenzung und den Übergang in einen Nachzustand denotieren, und in diesem Falle muß ein nicht-atomarer (gro $\beta$ werden) oder atomarer Übergang 
(schwanger werden) gleichermaßen erfaßt sein. Im im Lexikoneintrag wird nur die Invariante repräsentiert.

$$
\text { /werden/ } \quad \lambda \mathrm{P} \lambda \mathrm{x} \lambda \mathrm{s}[\mathrm{s} \text { INST }[\mathrm{CHANGE}[\mathrm{P} \mathrm{x}]]]
$$

CHANGE ist wie BECOME vom logischen Typ $<t, t>$; s (alias e) ist ein bezüglich des Typs noch unspezifisches Situationsargument.

Die Bedeutung von CHANGE richtig $\mathrm{zu}$ beschreiben, bereitet mir nach wie vor Kopfzerbrechen. Nach meinem jetzigen Verständnis umschreibe ich sie so:

- Die Bedeutung eines Ausdrucks ist mit s INST [CHANGE (Px)] zu repräsentieren genau dann, wenn CHANGE (Px) zur Situationszeit $T$ von $s$ gilt, und es keine Teilsituation $\mathrm{s}_{\mathrm{i}}$ von $\mathrm{s} z u$ einer Teilzeit $\mathrm{t}_{\mathrm{i}}$ von $\mathrm{T}$ gibt, in der die in $\mathrm{P}$ genannte Eigenschaft denselben Skalenwert hat.

- CHANGE ist eine gerichtete Veränderung, sie ist auf den Pluspol (oder Minuspol) einer Dimension / Eigenschaft oder auf einen Zustand gerichtet, die im Prädikat-Argument $\mathrm{P}$ genannt sind.

Im Stil von (4) formuliert hat CHANGE qua Wahrheitsbedingung die Bedeutung:

(6) $\quad \mathrm{s}$ INST $\|$ CHANGE $\|(P x)=1$, gdw. es gibt keine Teilsituationen $s_{j}$ und $s_{j}$ mit $s_{i}, s_{j} \subset s$ und $s_{j}{ }^{\circ} s_{j}$ derart, daß $P$ in $s_{i}$ denselben Skalenwert/Ausprägungsgrad hat wie $P$ in $s_{j}$.

$\left(\mathrm{s}_{\mathrm{i}}{ }^{\circ} \mathrm{s}_{\mathrm{j}}\right.$ für $\mathrm{s}_{\mathrm{i}}$ ist unmittelbar gefolgt von $\left.\mathrm{s}_{\mathrm{j}}\right)$

Diese Definition hat zwei Lesarten; die in den folgenden Abschnitten auszubuchstabieren sind:

1. Es gibt überhaupt kein $s_{j}$, für das $P(x)$ gilt. Auf keine Teilsituation von ,groß/schwanger werden" trifft ,groß/schwanger werden" zu, sondern nur auf die Gesamtsituation s.

2. Es gibt keine Teilsituationen $s_{i}, s_{j}$ mit gleichen Werten von $P$. Zwar trifft zu jeder Teilsituation $s_{i}, s_{j}$ einer Größerwerden-Situation $s$ auch "größer werden" $z u$, der Skalenwert auf der Dimension "Größe" differiert aber zu jedem $s_{i}$, $s_{j}$. In jeder Teilsituation $s_{j}$ ist der Skalenwert größer als in der vorausgegangenen $s_{\mathrm{i}}$.

Die Definition soll für alle nicht-statischen Prädikate mit der Bedeutungskomponente CHANGE gelten. Alle weitergehende Information ist davon abzuleiten. Die Bedeutung wird je nach Art des Prädikat-Arguments von werden angereichert mit einer spezifizierten Information über die der Komponente CHANGE inhärente Relation zur Zeit, über Nachbaroder Teilsituationen von s (s. Abschnitt 8.2).

Eine so angereicherte Repräsentation der Bedeutung von werden-Konstruktionen ist schließlich Basis für die Definition der Situationstypen, s. Abschnitt 8.3. Zum Vorverständnis sollen die für diesen Aufsatz geltenden Situationstyp-Bestimmungen vorgestellt werden 


\section{Vendlers Situationstypen}

Propositionen referieren qua Situationsargument $s$ in der Semantischen Form eines Verbs auf Situationen. Die Relevanz unterschiedlicher Situationstypen für die Grammatik steht nicht von vorn herein außer Frage. Sie muß sich erweisen und tut es etwa in Gestalt verschiedener syntaktischer Konstruktionen oder temporaler Modifikatoren, die s subkategorisieren. Es gibt Modifikatoren, die sich nur einem bestimmten Typ anschließen können, deren externes Argument also mit einem spezifischen Situationsargument des verbalen Modifikanden zu unifizieren ist. Die Kompatibilität von temporaler Adverbialklasse und verbalem Ausdruck ist mittlerweile eine anerkannte Testinstanz für die Zuordnung verbaler Ausdrücke zu Situationstypen, vgl. z.B. Herweg (1991b) und die Beispiele in Abschnitt 7.2.2.

Idealiter präformieren Verben als die lexikalischen Köpfe von Sätzen den Situationstyp. Sitzen ist ein Zustandsprädikat und charakterisiert zusammen mit seinen Argumenten eine Situation als homogen und statisch, wachsen ist ein Prozeßprädikat, altern, sterben und töten sind Ereignisprädikate, unabhängig von der Art eventueller Komplemente. Aber auch die Variabilität der Typzuordnung des Situationsarguments des Verbs ist in der Verbsemantik präformiert. Eine Gruppe von Verben, zu der die Bewegungsverben (laufen), die successive patient verbs (SUK-Verben in Krifka (1989)) essen und bauen und eben auch werden gehören, denotiert je nach Eigenschaft der Komplemente eine Situation vom Typ Prozeß oder Accomplishment.

Es ist zu betonen, daß nur Typzuordnungen der Art zur Debatte stehen, die spätestens als Resultat der Komposition von Verb- und Komplementbedeutung zustande kommen (wie bei essen, werden und laufen). Jeder so etablierte Situationstyp kann sekundär modifikatorisch relativiert werden, eine atelische Situation kann z.B. durch Zeitspannenadverbiale telisch werden, wie in drei Stunden arbeiten, eine telische Situation kann durch Iteration atelisch werden, vgl. stundenlang in Geschäfte rennen. Nur in diesem Fall sollte man von (teilweise durch Coercion zustandegekommener) sekundärer Typverschiebung sprechen.

$\mathrm{Da} ß$ unterschiedliche Situationstypen identifiziert werden können, muß nicht bedeuten, daß sie in den Lexikoneinträgen von Verben selbst repräsentiert sind. Mein Vorschlag läuft darauf hinaus, im Lexikoneintrag eines Verbs ein unspezifiziertes Situationsargument s anzunehmen, das durch geregelte Erweiterung des Ausgangsmaterials Basis einer Definition spezifischer Situationstypen ist.

Die Literatur in der Nachfolge von Vendler (1967) ist reich an Vorschlägen zur Kategorisierung und unterschiedlichen Gruppierung von Situationen und es gibt eine verwirrende terminologische Vielfalt. ${ }^{4}$ Ich nehme die vier Typen Zustand, Prozeß, Accomplishment und Achievement als gegeben und verstehe sie so:

Propositionen, die eine Situation als homogen beschreiben, enthalten keine Festlegung über einen Wechsel in einen anderen Zustand und damit auch keine Festlegung über einen komplementären Vorzustand. Nach Dowty (1979) sind stative Prädikate zeitlich homogen (divisiv). $\mathrm{P}(\mathrm{x})$ ist zum Intervall $\mathrm{T}$ wahr gdw. es ist zu allen Teilintervallen von $\mathrm{T}$ wahr ist.

Auch Propositionen, die Verbbedeutungen wie die von wachsen/größer werden, laufen enthalten, beschreiben wie die von wissen, sitzen, aber auch schlafen, sehen, eine Situation als homogen. Aber bei ersteren findet zu allen Teilintervallen von T eine Veränderung gleicher

\footnotetext{
4 Es gibt unterschiedliche Benennungen für den Begriff, neben Situations- oder Ereignistyp auch die anderweitig belegten Termini Aktionsart und Aspekt, was zu Mißverständnissen führt. Bis auf sich überschneidende Funktionsgebiete sind die grammatischen Kategoriengefüge perfektiver/imperfektiver Aspekt in den slavischen Sprachen, nonprogressive/progressive im Englischen usw., die über unterschiedliche Situationstypen operieren, sowie die Aktionsarten in Sprachen wie den slavischen oder finnougrischen, davon abzusetzen. Ich vermeide in diesem Aufsatz die Termini Aspekt und Aktionsart für den Begriff Situationstyp und benutze terminativ, telisch und inhomogen synonym für Eigenschaften von Ereignissen und entsprechend aterminitativ, atelisch und homogen für Eigenschaften von Zuständen und Prozessen.
} 
Art statt, der Wert der im Prädikat-Argument genannten Eigenschaft ändert sich in allen Teilsituationen $\mathrm{s}_{\mathrm{i}}$ von $\mathrm{s}$; wachsen, laufen beschreiben Prozesse. Bei letzteren ändert sich dagegen nichts, Peter schläft und Er sieht mich denotieren Zustände. Diese Festlegung unterscheidet sich von der gängigen, nach der Zustände (states) nicht-intendiert sind. ${ }^{5}$ Für Situationstyp-Distinktionen à la Vendler sind nach meinem Verständnis Prozeßhaftigkeit und Telizität, nicht aber Intentionalität relevant. Intentionalität (Agenshaftigkeit) ergibt eine zusätzliche Subklassifizierung sowohl von Zustands- (beobachten vs. sehen) wie von Ereignisbezeichnungen (töten vs. sterben).

Propositionen, die eine Situation als inhomogen beschreiben, enthalten dagegen Festlegungen über das anvisierte Ende der Situation, entweder - wie bei gequantelten IndividuenArgumenten (wie in ein Haus bauen) - darüber, daß das Objekt "nach und nach dem Ereignis unterzogen wird" oder - wie bei Prädikat-Argumenten (wie in groß werden oder ins Zimmer laufen) - darüber daß ein anvisierter Nachzustand zu erreichen ist. Im letzteren Fall ist ein Zustandswechsel involviert, Vor- und Nachzustände sind komplementär zu einander und jeweils zu unterschiedlichen Zeiten wahr.

Ein Kriterium, Zustände und Prozesse zu diskriminieren, ist die mögliche Alternation von Atelizität und Telizität. Nur Prozeßverben wie laufen und bauen (also die Bewegungs- und SUK-Verben) bilden mit einem geeigneten Argument ein telisches Prädikat (in die Stadt laufen, einen Apfel essen), nicht aber Zustandsverben wie beobachten (die Kinder beobachten), sie bleiben immer atelisch.

Verben mit prozessualen Eigenschaften können mit einer Zielangabe oder einer gequantelten DP eine Konstruktion bilden, die einen zeitlich ausgedehnten oder zumindest ausdehnbaren Übergang zu einem Nachzustand denotiert (in die Stadt laufen, ein Haus bauen). Nur solche Prädikate vom Subtyp Accomplishment haben ein atelisches Pendant (südwärts laufen, Brei essen). Dagegen ist der Zustandswechsel beim Subtyp Achievement von Ereignissen (ankommen, finden) ein atomarer, ohne zeitliche Ausdehnung; diese Verben bleiben Achievements in allen Komplement-Kontexten (einen Groschen:Groschen finden), es sei denn eine nichttelische Lesart wird erzwungen.

In erster Annäherung sollen "Prozeßhaftigkeit" und "Telizität" die unterschiedlichen Situationstypen bestimmen.

\section{(7) Situationstypen}

\begin{tabular}{|c|c|c|c|c|}
\hline & Verben & prozeßhaft & telisch & Situationstyp \\
\hline 1 & $\begin{array}{l}\text { groß/wach sein, schlafen, } \\
\text { im Zimmer stehen, wissen, } \\
\text { jemanden sehen }\end{array}$ & . & - & Zustand \\
\hline 2 & $\begin{array}{l}\text { größer werden, wachsen, } \\
\text { schweifen,westwärts gehen } \\
\text { Häuser bauen, Wein trinken }\end{array}$ & + & - & Prozeß \\
\hline 3 & $\begin{array}{l}\text { groß werden,altern,verarmen, } \\
\text { in die Stadt gehen } \\
\text { ein Haus bauen }\end{array}$ & + & + & $\begin{array}{l}\text { Ereignis } \\
\text { (Accomplishment) }\end{array}$ \\
\hline 4 & $\begin{array}{l}\text { sterben, schwanger werden } \\
\text { ankommen, betreten, } \\
\text { einen Groschen finden }\end{array}$ & - & + & $\begin{array}{l}\text { Ereignis } \\
\text { (Achievement) }\end{array}$ \\
\hline
\end{tabular}

\footnotetext{
${ }^{5}$ Nach Dowty (1979) sind be tall, be true; exist, stink, live, burn; like, similar, proud; love, know, see, hear, be in NP, sit in NP... Statives mit den bekannten syntaktischen Besonderheiten (keine progressive form im Englischen, kein Imperativ usw.). Activities (volitional) sind bei ihm: be brave, be nice to NP; be a clown; run, swim; rain; eat; drive, push NP; seek for; watch...
} 
laufen und werden wechseln zwischen zwei unterschiedlichen Typen, aber werden kommt als einziges Verb in allen drei nicht-statischen Situationstypen Prozeß, Accomplishment, Achievement vor, das ist der Ausgangspunkt meiner Analyse.

\section{Maximale Unterbestimmtheit contra telische Bedeutung von werden}

In den eingangs zitierten Beispielen
a) groß werden : nach Berlin gehen
b) größer werden : südwärts gehen
c) schwanger werden: in Berlin ankommen

ist die Telizität der Sätze in (a) und (c) nicht Gegenstand der Kontroverse. Auch die Atelizität der Sätze in (b) kann angesichts der bekannten Tests mit temporalen Adverbialen unterschiedlicher Subklassen nicht bestritten werden, vgl. die Beispiele (34) in Abschnitt 7.2.2. Uneins bin ich mit meinen Kontrahenten aber darin, wie in größer werden die Atelizität zustande kommt und folglich auch in der Deutung der Kopula werden.

Meine Annahmen:

- Eine Adjektivphrase wie groß, schwanger (natürlich auch ein nominales Prädikativ wie Lehrer) denotiert im Kontext von werden den Ziel- oder Nachzustand einer Veränderung. Diese Adjektive bezeichnen inhärent oder durch Bezug auf eine Vergleichsphrase, einen Normwert, einen festen Wert einer Dimension oder eine Eigenschaft.

- Peter wird groß und Anna wird schwanger sind somit dank der Komplemente telisch, ein vorausgesetzter komplementärer Vorzustand kann aus der Proposition errechnet werden.

- Einer AP im Komparativ wie größer fehlt dagegen der Bezug auf einen festen Normwert, sie ist auf einen variablen Vergleichswert bezogen.

- Größer werden ist anders als größer sein nicht notwendig elliptisch. Wenn nicht wenigstens der Kontext den Vergleichwert liefert (Anna wurde größer (als Karl)), ist die denotierte Veränderung atelisch, es lassen sich keine Angaben über einen zu erreichenden Zielzustand rekonstruieren.

- Eine atelische Veränderungssituation wird als Prozeß interpretiert. Die dem Komparativ inhärierende Vergleichsinstanz ist die Zeit, der Fortgang der Veränderung und der Fortgang der Zeit werden parallelisiert.

- Prozesse wie Peter wird größer und Zustände wie Peter ist groß/größer als Paul enthalten keine Angaben über Beginn oder Ende einer Situation, weder Ziel- noch vorausgesetzten Vorzustand. Genau darin unterscheiden sich beide von Ereignissen.

- Prozesse und Ereignisse haben durch das Veränderungsprädikat CHANGE einen impliziten Bezug zum Vergehen der Zeit und sind insofern gerichtet.

Ich will das im Einzelnen erläutern:

Die AP-formatigen Komplemente von werden variieren über drei Subklassen. Die drei Realisierungsarten von $\mathrm{P}, \mathrm{P}_{\text {relk }}, \mathrm{P}_{\text {relp }}$ und $\mathrm{P}_{\mathrm{abs}}$, sind nach ihren lexikalischen Köpfen benannt:

$$
\begin{array}{ll}
\text { größer } & \mathrm{P}_{\text {relk }}: \text { relative Adjektive im Komparativ: } \\
\text { groß } & \mathrm{P}_{\text {relp }} \text { : relative Adjektive im Positiv } \\
\text { schwanger } & \mathrm{P}_{\text {abs }} \text { : absolute Adjektive }
\end{array}
$$


Bezüglich der Dimensionsadjektive folge ich den Bedeutungsrepräsentationen in Bierwisch (1987) und Zimmermann (1998a) :

\section{$5.1 \quad$ Relative Adjektive $\left(P_{\text {rel }}\right)$}

Relative Adjektive sind skalierbar, sie bezeichnen Eigenschaften, deren unterschiedliche Ausmaße Werten auf einer Skala entsprechen. Sie sind inhärent auf eine Vergleichsklasse bezogen.

\section{Relative Adjektive im Positiv $\left(\mathbf{P}_{\text {relp }}\right)$}

Gro $\beta$ ist ein zweistelliges Prädikat und denotiert das Ausmaß der maximalen Dimension "Größe" eines Objektes in Bezug auf eine Vergleichsinstanz. In Anlehnung an Bierwisch (1987:150) und Lang (1987: 431ff) sei groß im Positiv wie in (9) repräsentiert",

$$
/ g r o \beta /(\lambda \mathrm{c}) \lambda \mathrm{x}[\mathrm{QUANT} \text { SIZE } \mathrm{x}=\mathrm{v}+\mathrm{c}]
$$

SIZE in der SF von gro $\beta$ ist ein Parameter, der kontextabhängig für die jeweiligen Dimensionen von x spezifiziert wird; QUANT projiziert SIZE x auf die entsprechende Skala. Die relativen Dimensionsadjektive sind auf eine Vergleichsinstanz bezogen, sie enthalten einen weiteren Parameter $\mathrm{v}$, dessen Wert 0 oder $\mathrm{N}_{\mathrm{c}}$ durch eine Vergleichsklasse festgelegt ist; $\mathrm{c}$ steht für den Differenzbetrag, der fakultativ durch eine Gradphrase spezifiziert und bei + PolAdjektiven dem Vergleichswert hinzugefügt wird.

Bei komplementlosem Adjektiv ist die Vergleichsinstanz eine Klasse von Objekten $\mathrm{N}_{\mathrm{c}}$ mit bezüglich der Größe durchschnittlichem Wert. Sie ist i.A. rekonstruierbar, bleibt also implizit. In diesem Fall muß $\mathrm{c}$ existentiell gebunden sein. Die Vergleichsklasse ist aber spezifizierbar, wie (10) zeigt:

\section{Peter war groß (für sein Alter / für einen Kater / verglichen mit anderen Kindern)}

Ergänzt von einer Gradphrase wie 1,60 $\mathrm{m}$ gro $\beta$ hat $\mathrm{v}$ den Skalenwert $0, \mathrm{c}$ den Wert $1,60 \mathrm{~m}$ :

$$
\text { Peter war 1,60 groß (also klein für sein Alter). }
$$

Relative Adjektive im Komparativ $\left(P_{\text {relk }}\right)$

In dieser Komparationsform ist die Vergleichsinstanz komplexer, in der Notation von Zimmermann (1998a):

$$
\text { lgrößer/ } \quad(\lambda \mathrm{c})(\lambda \mathrm{W}) \lambda \mathrm{x} \forall \mathrm{c}^{\prime}\left[\mathrm{Wc} \rightarrow\left[\text { QUANT DIM } \mathrm{x}=\mathrm{c}^{\prime}+\mathrm{c}\right]\right]
$$

-er verwandelt ein zweistelliges in ein dreistelliges Prädikat. Der Komparativ hat keinen Normbezug, er referiert auf einen variablen Wert c, dessen Bezugsinstanz allein der Vergeichswert c' ist, der Wert einer Vergleichsphrase W.

(13) a) Er ist größer als die Eltern (groß sind) / als ich gedacht habe (daß er groß ist) b)Der andere Tisch ist länger

Für relative Adjektive gilt also:

\footnotetext{
${ }^{6}$ Auch Bewertungsadjektive sind graduierbar. Bei diesen bezieht sich die Graduierbarkeit auf den Ausprägungsgrad der denotierten Eigenschaft, bei Dimensionsadjektiven auf das Ausmaß der jeweils spezifizierten Dimension. Ich beschränke mich auf die Dimensionsadjektive als Demonstrationsobjekte.
} 
- Relativen Adjektiven (hier Dimensionsadjektiven) inhäriert eine Vergleichsinstanz, die durch Gradphrasen wie 10 Jahre, $2 \mathrm{~m}, 5 \mathrm{~kg}$, sehr spezifiziert werden kann.

- Sie sind mindestens zweistellig.

- Der Positiv eines Dimensionsadjektivs ohne Komplement ist immer normbezogen. Es nimmt den Normwert als Vergleichswert und bezeichnet einen festen Abschnitt oberhalb (bzw. unterhalb) von diesem.

- Relative Adjektive haben neben der Positivform typischerweise eine Komparativform.

- Der Komparativ ist nie auf einen Normwert, sondern auf einen beliebigen anderen Vergleichswert W bezogen. Aus dem unterschiedlich instantiierbaren Vergleichswert W resultiert der variable Wert des Komparativs.

- AP im Komparativ + sein ohne explizite Vergleichsinstanz kann nur als Ellipse interpretiert werden.

- Die Antonyme (z.B. klein : groß) stehen in konträrer Relation zueinander, wenn „klein“ nicht wahr ist, muß deshalb nicht ,groß“ wahr sein.

\subsection{Absolute Adjektive $\left(\mathbf{P}_{\text {abs }}\right)$}

Absolute Adjektive sind nicht auf eine Vergleichsinstanz bezogen, sie sind einstellige Prädikate (vgl. auch E. Klein (1980), von Stechow (1983)), vgl. den Lexikoneintrag von schwanger:

\section{(14) /schwanger/ $\lambda \mathrm{x}[\mathrm{SCHWANGER} \mathrm{x}]$}

Daraus leiten sich weitere Eigenschaften absoluter Adjektive ab:

- Sie sind i.A. nicht durch Gradphrasen modifizierbar.

- Sie haben typischerweise keine Komparativform. Wenn ein absolutes Adjektiv wie krank, schwarz im Komparativ steht, dann geht es nur um ein unterschiedliches Ausmaß innerhalb der Domäne der Eigenschaft "krank, schwarz".

- Die antonymen Ausdrücke lebendig : tot, nicht schwanger : schwanger stehen in kontradiktorischer Relation zu einander, wenn „schwanger“ nicht wahr ist, dann muß ,nicht schwanger" wahr sein.

\section{Kontextabhängige Spezifizierung von CHANGE}

Sein beschreibt statische Situationen unabhängig von der jeweiligen Spezifikation der Prädikatenvariablen P als AP im Positiv oder Komparativ, als DP oder als PP.

(15) groß/größer/schwanger sein

Lehrer sein

im Zimmer sein

Werden beschreibt unabhängig von der Art seiner Komplemente nicht-statische oder Veränderungssituationen. Eine Veränderungssituation ist von einer unmarkierten statischen Situation unterschieden durch das Prädikat CHANGE. Sie ist aber zunächst offen bezüglich Telizität und/oder Prozeßhaftigkeit der Veränderung. Die Festlegung dieser Charakteristika muß in der Bedeutung der drei Subklassen des Prädikat-Arguments begründet sein.

$\begin{array}{lll}\text { größer werden } & \mathrm{P}_{\text {relk: }}: & \text { Proze } \beta \\ \text { groß werden } & \mathrm{P}_{\text {reip }}: & \text { telisch, Proze } \beta \\ \text { schwanger werden } & \mathrm{P}_{\text {abs }}: & \text { telisch }\end{array}$


Veränderung ist - unabhängig vom im Satz gewählten Tempus - notwendig auf die Zeit bezogen.

- Zeitbezug ist der Beitrag von werden zur Konstruktion AP + werden.

- Die Art der Relation von Veränderungs- und Zeitablauf hängt dagegen von den unterschiedlichen Spezifizierungen des Prädikat-Arguments $\mathrm{P}$ ab. Werden in Kombination mit $\mathrm{P}_{\text {relk }}, \mathrm{P}_{\text {relp }}$ oder $\mathrm{P}_{\text {abs }}$ erzeugt unterschiedliche Bezugnahmen der Situation s auf die Zeit: Bei $P_{\text {relk }}$ auf Teilsituationen von $s$ zur Situationszeit $T$ (gekennzeichnet durch unteren Index $s_{i}$ ), bei $P_{\text {relp }}$ und $P_{a b s}$ auf die Nachbarsituationen vor und nach $T$ (gekennzeichnet durch oberen Index s', $\left.s^{\prime \prime}\right)$.

\subsection{Relative Adjektive + werden}

Relative Adjektive sind uneingeschränkt mit werden kombinierbar.

Die Eigenschaft relativer Adjektive, in konträrer Beziehung zu ihrem Antonym zu stehen, wirkt sich im Kontext von werden folgendermaßen aus (vgl. auch Steinitz (1975:40-44):

Relative Adjektive + werden beruhen auf einem Kontinuum-Konzept. Gro $\beta$ werden schließt für einen Ausgangszustand ,nicht groß“ eine Zunahme von Werten in Richtung „groß“, also ein Größerwerden ein; die Übergangsphase zum Nachzustand ist bei relativen Adjektiven also ausdehnbar. Es ist sprachlich gesehen irrelevant, daß sie de facto beliebig zusammenschrumpfen kann, wie in Er knipste das Licht aus und es wurde dunkel.

- Die Ausdehnbarkeit der Übergangsphase ist Basis für den möglichen Wechsel zwischen telischer und atelischer Situation.

\subsection{1 größer werden}

Die Komposition der Bedeutungen von werden und größer ergibt:

$$
\left.\lambda \mathrm{x} \lambda \mathrm{s}\left[\mathrm{s} \text { INST [CHANGE } \forall \mathrm{c}^{\prime} \exists \mathrm{W} \exists \mathrm{c}\left[\mathrm{Wc}^{\prime} \rightarrow\left[\text { QUANT DIM } \mathrm{x}=\mathrm{c}^{\prime}+\mathrm{c}\right]\right]\right]\right]
$$

Bierwisch (1987:179) meint in einer Nebenbemerkung, der komplementlose Komparativ in Peter wird größer sei wie bei Peter ist größer als Ellipse zu interpretieren, mit einer Proform für das Komplement - die Variable W für die Vergleichsphrase - rekonstruierbar etwa durch als vorher. Von einer Ellipsen-Analyse gehen auch von Stechow (1984) und Heim (1985) aus. Ich halte diese Erklärung für unzutreffend. Peter wird größer kann nur dann analog zu Peter ist größer interpretiert werden, wenn aus dem Kontext eine Vergleichsinstanz wirklich erschließbar ist, wie in

(Ich hatte gedacht, Peter wird so wie seine Eltern, aber) Peter wird größer (als sie) (Ich hatte gedacht, daß Peter so bleibt wie er zu Beginn unserer Bekanntschaft war, aber) Peter wurde größer (als zu Beginn / als er vorher war)

Die möglicherweise implizite Bezugnahme auf eine solche Vergleichsinstanz ist aber bei größer werden - anders als bei größer sein! - nicht obligat, sie ist nicht einmal der Normalfall. Beim zu größer werden synonymen synthetischen Verb wachsen ist sie sogar unmöglich, vgl..

Peter wurde größer $=$ Peter wuchs

(20) Peter wurde größer als seine Eltern / als er vorher war

Peter *wuchs als seine Eltern / als er vorher war 
Sätze wie (19) sind nicht elliptisch zu verstehen, die Vergleichsinstanz ist nicht Peters Größe $\mathrm{zu}$ einer zu bestimmenden Vorzeit, so wenig wie in Abends lief Peter der denotierte Prozeß auf den Beginn der Bewegung Bezug nimmt. Relativen Adjektiven inhäriert aber per definitionem eine Vergleichsinstanz. Welche ist das bei (19) gegenüber (20)?

Die vergehende Zeit ist als Bezugsgröße immer verfügbar. Den Zeitbezug trägt in unserem Fall werden bei, CHANGE in der SF von werden steht für unspezifische Veränderung in der Zeit. Zusammen mit werden gibt das Adjektiv im Komparativ die Richtung der Veränderung eines im Subjekt genannten Individuums an, ohne jedoch eine Begrenzung für die Veränderung auszudrücken.

- Eine Veränderung ohne Begrenzung ist ein Prozeß.

Der Unterschied zwischen (19) und (20) wird deutlich bei modifikatorischer Erweiterung durch Zeitdauer- oder Zeitspannenadverbiale:
a) (Ich hatte gedacht Peter wird so groß wie seine Eltern, aber) er wurde *jahrelang / in einem Jahr größer (als sie)
b) Peter wurde jahrelang größer $\left({ }^{*}\right.$ als seine Eltern $)$

Peter wird größer in der Lesart von (21a) ist telisch. Auch wenn die Vergleichsklasse nur aus dem Kontext zu erschließen ist, legt sie einen Nachzustand fest, wo Peter größer als sie ist, der Satz ist ein Accomplishment. (21b) hat keinen aus dem Kontext erschließbaren Vergleichswert, ist also atelisch. Werden + Adjektiv im Komparativ ist in diesem Sinne ambig, vgl. auch

\section{Die Musik wurde lauter / besser}

Entweder der Kontext liefert einen Vergleichs- und deshalb spezifischen Wert (telische Situation) oder wie haben einen unspezischen, sich verändernden Wert.

Ein Adjektiv im Komparativ nimmt den Inhalt der jeweiligen Vergleichsphrase W als Vergleichswert. Bei größer werden steht im Default-Fall, d.h. wenn keine spezielle Vergleichsinstanz rekonstruierbar ist, die Zeitachse zur Verfügung. Im Kontext von werden wird dann $\mathrm{W}$ durch ein Zeitintervall $\mathrm{T}$ instantiiert, der Zuwachsindex der Eigenschaft kovariiert mit dem Zeitindex. Wie bei homogenen Situationen generell gilt zwar zu jedem Teilintervall $t_{j}$ eines Zeitintervalls T dieselbe Proposition CHANGE Px, aber der Wert von $\mathbf{P}$ ist variabel. Der Wert der Eigenschaft „Größe“ eines Individuums $z u t_{i+1}$ übersteigt den Wert $c^{\prime}$ derselben Eigenschaft $\mathrm{zu} \mathrm{t}_{\mathrm{i}}$ um einen Differenzbetrag c.

Ausgenommen eine als tautologisch empfundene Paraphrase wie Hans wird mit der Zeit größer, ist die Vergleichsinstanz Zeit im Kontext von werden syntaktisch nicht zu realisieren.

\subsection{2 groß werden}

Bei gro $\beta$ werden ist dagegen eine Begrenzung der Veränderung durch die positive Form des Adjektivs enthalten, gro $\beta$ referiert auf einen festen Abschnitt $\mathrm{c}$ jenseits des Normwertes $\mathrm{v}$ einer Vergleichsklasse. Im Kontext von werden wird dieser Abschnitt als Ziel der Veränderung interpretiert. Mit dem Überschreiten des Normwertes geht die Veränderung in einen Nachzustand "groß sein" über. Eine Veränderung mit einem implizierten Nachzustand ist ein Ereignis. ${ }^{7}$ Der Gesamtausdruck ist telisch. Basis dieser Interpretation ist die Komposition der SF von groß und werden:

\footnotetext{
${ }^{7}$ Genauso würde ich mit Resultativkonstruktionen wie das Glas leer trinken verfahren. Weder in der Bedeutung des Verbs noch in der seines Komplements kann BECOME verankert sein. "Trinken" ist ein Veränderungsprädikat, in dessen Kontext wird das Zustandsprädikat "das Glas (ist) leer" als Nachzustand interpretiert. Aber das ist eine andere Geschichte.
} 


\subsection{Absolute Adjektive + werden}

Die Eigenschaft absoluter Adjektive, in kontradiktorischer Beziehung zu ihrem Antonym zu stehen, wirkt sich im Kontext von werden in folgender Weise aus (vgl. auch Steinitz (1975:40-44): Absolutes Adjektiv + werden beruht auf dem Konzept zweier diskontinuierlich aufeinander folgender Zustände. Schwanger werden involviert zwei kontradiktorische Eigenschaften/Zustände, den Vorzustand ,nicht schwanger" und den Nachzustand "schwanger", die zeitlich unmittelbar aneinander grenzen. Das Situationsargument s des Ausdrucks bezieht sich genau auf diese Grenze, es ist ein punctual event, also atomar. Nur im jüdischen Witz kann man ein bißchen schwanger werden. Der atomare Wechsel zeichnet Achievements gegenüber Accomplishments aus. ${ }^{8}$

\section{/schwanger werden/ $\lambda \mathrm{x} \lambda \mathrm{s}$ [s INST [CHANGE [SCHWANGER $\mathrm{x}]]$}

Relative Adjektive + werden alternieren je nach Form ihres Komplements zwischen Accomplishment und Prozeß. Ereignisverben mit einem absoluten Adjektiv als Basis sind dagegen auf den einen Situationstyp Achievement festgelegt. Denselben Unterschied finden wir bei den Bewegungsverben, dort allerdings auf zwei unterschiedliche Lexeme verteilt: laufen : eintreten, ankommen, (vgl. (südwärts) laufen/ins Zimmer laufen: ins Haus eintreten). Wenn absolute Adjektive eine Komparativform haben, wie krank : kränker, und mit werden kombinierbar sind, verhalten sie sich anders als relative Adjektive. Die Sätze in (27a) sind semantisch einwandfrei, nicht aber die in (27b); wer kränker wird, ist schon krank, wer dicker wird, muß nicht schon dick sein:

a)Peter wird dick: Er wird dicker und (bald) dick sein

Peter wird dicker, aber nicht dick

b) Peter wird krank: *Er wird kränker und (bald) krank sein Peter wird kränker, *aber nicht krank

Für absolute Adjektive gibt es auffallende, aber noch nicht geklärte Selektionsbeschränkungen im Kontext von werden. Absolute Adjektive wie nackt, kaputt, offen, tot, frei denotieren zusammen mit der Kopula sein Zustände, genauso wie relative Adjektive. Zur Bezeichnung von Veränderungssituationen stehen diese Adjektive aber nicht als analytische, sondern nur als synthetische Konstruktion oder mit einem Bewegungsverb kombiniert zur Verfügung, vgl.

a) zerbrechen, sich öfnen/aufgehen, sterben, freikommen, pleitegehen

b) *nackt /*kaputt/*offen /* tot / *frei werden, *pleite werden

Lange Zeit nahm ich diesen Befund, den viele Sprachen teilen, als Beleg dafür, daß die Bedeutung von werden eine prozessuale Komponente hat, was eine Kombination mit absoluten Adjektiven ausschließt. Es gibt aber viel mehr Gegenbeispiele, als ich zunächst mit schwanger/volljährig/krank werden angenommen hatte. Amrhein (1999) hat aus verschiede-

\footnotetext{
${ }^{8}$ Dem widerspricht nur scheinbar, daß Achievementverben im Russischen den imperfektiven Aspekt haben können (das Deutsche hat nur die Möglichkeit lexikalischer Umschreibungen), vgl. umirat': im Sterben liegen. Aber umirat' ist nicht im gleichen Sinn atelisch wie wachsen, hier haben wir es mit einer sekundären Situationstyp-Verschiebung zu tun, eine der Funktionen von Aspekt. Achievement-Verben unterscheiden sich von Momentanverben wie blitzen, knallen dadurch, daß bei ihrem Denotat Vor- und Nachzustand kontradiktorisch zu einander sind. Bei Momentanverben stimmen Vor- und Nachzustand überein.
} 
nen Quellen eine ganze Batterie von werden-Konstruktionen zusammengestellt, die Achievements denotieren, eine Auswahl:

(26) In Hessens rot-grüner Landesregierung werden demnächst zwei Stellen frei. ... wird es ... beinahe selbstverständlich, sich oben ohne porträtieren zu lassen Seither ist Nacktheit ungefährlich geworden.

Der Traum wird nicht wahr werden.

\section{Der Streitfall größer werden}

Nach Darlegung meiner Position kann ich mich auf den Diskurs mit der übermächtigen Gegenposition, Auffassung A, im Streitfall größer werden einlassen.

\subsection{Auffassung A:}

Meine Kontrahenten haben sich dafür entschieden, daß werden ein telisches Verb ist. Dabei ist es unerheblich, ob BECOME Px als "a nearly punctual transition" (Wunderlich (1994)) oder als Accomplishment interpretiert wird (von Stechow (1996), Musan (1999)) ${ }^{9}$.

Der Prozeßcharakter, den größer werden denotiert, ist bei allen Proponenten der Auffassung A abgeleiteter Natur. Der ,scheinbare Prozeß-Charakter kann nur aufgrund eines sekundären Effekts zustandekommen." (Musan (1999:4). Musan (ibid:15) meint "Für den Prozeßeffekt bei größer werden (ist) eine durch den Komparativ eingebrachte Iterativitätskomponente verantwortlich." Von Stechow (1998) schreibt unter Verweis auf von Stechow (1996), "Der Satz Das Barometer fällt (ist) zum Intervall $t$ wahr, wenn $t$ ein Werden ist mit dem Resultat, daß das Barometer tiefer steht als am Anfang von t. Die Aussage ist atelisch ("ein Prozeß"), und trotzdem haben wir den BECOME-Operator für "Accomplishments" vorliegen. Mit anderen Worten, es gibt Propositionen, die "Accomplishments" sind und trotzdem die Teilintervalleigenschaft haben. Deswegen spricht Dowty ja von degree achievements." Analog zu fallen in von Stechow (1996:125) beschreibt er größer werden:

$$
\left.\lambda x\left[\operatorname{BECOME}(\mathrm{t})\left(\lambda \mathrm{t}^{*} \cdot \operatorname{MORE}\left[\lambda \text { d.d-gro } \beta_{\mathrm{t}}^{*}(\mathrm{x}), \lambda \mathrm{d} \cdot \mathrm{d}-\mathrm{gro} \beta_{\mathrm{beg}(\mathrm{t})}(\mathrm{x})\right]\right)\right)\right]
$$

mit der Semantik des "abstrakten Komparativmorhems" MORE nach Seuren (1973):

$$
\| \text { MORE } \|(P, Q) \text { iff } \exists \mathrm{d}[\mathrm{P}(\mathrm{d}) \& \neg \mathrm{Q}(\mathrm{d})] \text {, where } \mathrm{P} \text { and } \mathrm{Q} \text { are properties of degrees. }
$$

Alle Details beiseitegelassen, enthalten in dieser Darstellung Prozeß-Bezeichnungen sowohl Anfang (Index "beg(t)") wie Ende oder Resultat (in BECOME angelegt) einer Situation, also die Ingredienzien eines telischen Prädikats.

\section{Einwände:}

Die Annahme, daß atelische Prozesse wie größer werden aus telischen Ereignissen mit der Bedeutungskomponente BECOME abgeleitet sind, hat weitreichende Folgen.

1. Prozeß, eigentlich einer der vier grundlegenden Situationstypen, wird $\mathrm{zu}$ einem durch sekundäre Modifikation (Iteration) entstandenen Typ degradiert. Das läuft hinaus auf eine Gleichsetzung von Sie wurde jahrelang dicker mit der durch das Adverbiale erzwungenen

\footnotetext{
9 Musan (1999:3) schreibt "Accomplishments (lassen) als situationsbedingten Sonderfall auch punktuelle Übergänge zu." Damit soll sowohl das plötzliche Reichwerden wie das Schwangerwerden als Sonderfall von Accomplishments eingeordnet sein.
} 
iterativen Lesart in Sie radelte jahrelang zum Bahnhof oder in Wie lange entdeckte sie Formeln?, Maria nieste letzten Sommer in Musan (ibid:15, 18). Vgl. dazu auch Abschnitt 7.2.1, Innere vs. äußere Iteration.

2. Konsequenterweise müßten z.B. die Bewegungsverben laufen, fahren usw., die wie werden zwischen Prozeß und Accomplishment alternieren, in ihrer SF eine telische Komponente enthalten. Der prozessuale Charakter wäre dann wie bei werden abgeleitet. Laufen müßte also ein telisches Verb der Ortsveränderung sein und Peter läuft (abends stundenlang) als Folge wiederholter Ortsveränderungen repräsentiert werden. Natürlich tut das niemand.

3. Bei einer Veränderung ohne Begrenzung stimmt es nicht, daß $\neg \mathrm{Px}$ aufhört und die komplementäre Eigenschaft Px anfängt zu gelten (vgl. Musan (1999:6/7), das hieße jeweils feste Werte wie bei groß werden annehmen. Bei größer werden - wie bei homogenen Situationen allgemein - ist aber weder ein Vor- noch ein Nachzustand involviert. Was sich ändert, ist lediglich der Skalenwert der Dimension „Größe“.

Jackendoff (1996:316f) nennt die Annahme von Auffassung A ein 'snapshot'-Verfahren, das den Tatbestand aus folgenden Gründen nicht richtig wiedergibt:

1. "(...) it misrepresents the essential continuity of events of motion. ... The choice of a finite set of subevents is altogether arbitrary." 2. "A finite sequence of subevents necessarily has a specified beginning and ending, so it cannot encode the absence of endpoints. Thus encoding nonbounded events requires major surgery in the semantic representation." 3. "It does not say that in each of these snapshots the object is moving, rather than suddenly appearing at a new location in the new subevent ... like an old-time movie, flickering from one state to another."

Ein Vorschlag von Jäger (in Vorbereitung) zur Definition von CHANGE verfährt gerade umgekeht, Ereignisse sind eine Art degenierter Prozesse. CHANGE hat qua Wahrheitsbedingungen bei ihm die Bedeutung

$\|$ CHANGE $\|(\mathrm{P})(\mathrm{s})=1 \mathrm{gdw}$. für jede Teilsituation s' von s gilt: Der Ausprägungsgrad von $\mathrm{P}$ ist im Vorzustand von s' geringer als im Nachzustand von s'

Dieser Definition liegt die Intuition zugrunde, daß alle nicht-statischen Prädikate eine Veränderung entlang einer bestimmten Skala denotieren. Alle diese Prädikate enthalten den Bedeutungsbaustein CHANGE, und die jeweilige Skala wird durch das Argument "P" determiniert. Bei Accomplishments und Achievements degeneriert diese Skala $\mathrm{zu}<$ nicht $\mathrm{P}$, $\mathrm{P}>$, und das traditionelle BECOME ergibt sich als Spezialfall von CHANGE. Im Falle von Prozessen wie größer werden ist diese Skala nicht-degeneriert und wir erhalten eine ausgedehnte Veränderung ohne festgelegten Zielpunkt, also ein atelisches Prädikat.

Nach meiner Auffassung ist aber werden unterspezifiziert sowohl bezüglich der Telizität wie der Prozeßhaftigkeit einer Situation. Beide Spezifikationen ist Sache der Komplemente der Kopula.

\subsection{Auffassung B:}

Jackendoff (ibid.): "Instead of treating moving as a finite sequence of states, I would like to encode it as continuous change over time. (...) for any arbitrary moment of time, there is a corresponding position."

Kaufmann (1995:43f) formuliert die Eigenschaften von Prozeßverben korrespondierend mit Wunderlich / Herweg (1991) ähnlich: "Prozesse sind wie Zustände homogen und zeitlich nicht begrenzt. (...) Da Prozesse eine Veränderung beinhalten, können sie durch monotone Funktionen aus der Zeit in eine verbspezifische Dimension charakterisiert werden, an der sich 
die Veränderung manifestiert. Diese 'verbspezifische Dimension' kann unterschiedlichen Charakter haben: Bei Verben wie essen bei denen der Vorgang eine Abarbeitung des vom Objekt bezeichneten Thema-Arguments beinhaltet, stellt das Objekt ... die Dimension.... Im Fall der BV liefert diese in MOVE kodierte Abbildung den im Raum zurückgelegten Weg: Zu unterschiedlichen Zeitpunkten nimmt das sich bewegende Objekt unterschiedliche Regionen ein, die den Weg konstituieren."

Meine im vorangegangenen Abschnitt vorgestellten Annahmen stimmen mit dieser Auffassung überein. Da sich die meisten meiner Opponenten an diesem Punkt festbeißen, möchte ich noch einmal den Unterschied herausstreichen zwischen einer AP mit einem relativen Adjektiv im Positiv $\left(\mathrm{P}_{\text {relp }}\right)$ und einem im Komparativ $\left(\mathrm{P}_{\text {relk }}\right)$ als Kopf:

Beide sind auf Vergleichswerte bezogen, aber das Adjektiv im Positiv nimmt einen Normwert als Vergleichswert und bezeichnet einen Abschnitt oberhalb (für groß) bzw. unterhalb (für klein) von diesem. Im Kontext von werden bekommt es den Status eines Ziels oder Nachzustanđs der Veränderung. Das Adjektiv im Komparativ nimmt den Inhalt der jeweiligen Vergleichsphrase W als Vergleichswert. Im Kontext von werden ist im uns hier interessierenden Default-Fall $\mathrm{W}$ durch ein Zeitintervall $\mathrm{T}$ instantiiert, dessen Teilintervalle $\mathrm{t}_{\mathrm{i}}$ mit den Teilsituationen $\mathrm{s}_{\mathrm{i}}$ von s pararallelisiert werden. Kein Ziel ist in Sicht. Größer werden denotiert zwar eine Veränderungs-, aber keine Zustandswechsel-Situation.

\section{WeitereArgumente für Auffassung B:}

\subsubsection{Innere vs. äußere Iteration}

Nach Auffassung A bezieht sich das Situationsargument $\mathrm{s}$ von werden aufgrund der Eigenschaften von BECOME zunächst auf ein telisches Ereignis. Atelizität kommt in den folgenden Sätzen unterschiedslos durch die Annahme einer nichtlimitierten Wiederholung eines solchen Ereignisses zustande.

a)Peter wurde (jahrelang) dicker / nahm (jahrelang) zu

b) Die Soldaten töteten (wochenlang) Geiseln

c) Peter fuhr einen Sommer lang mit dem Rad auf die Datsche

Er betrat wochenlang das Zimmer ohne zu grïßen

Maria nieste von zehn bis zwölf Uhr

Ich sehe folgende Unterschiede zwischen den Sätzen:

(30a): Die Bedeutung von zunehmen alias dicker werden enthält keine Festlegung über Grenze oder Nachzustand der Veränderung. Bei Abwesenheit der Begrenzung einer Veränderung gilt diese als mit der Zeit fortfahrend. Es sind dann Elementarsituationen $s_{i}$ parallel $\mathrm{zu}$ Zeitpunkten $t_{i}$ auszumachen, so daß Peter $\mathrm{zu}$ jedem $t_{\mathrm{i}}$ dicker ist als $\mathrm{zu}$ einem früheren. Merke wohl, dies ist eine Interpretation von CHANGE in einem bestimmten Kontext.

(30b): töten dagegen enthält in seiner Bedeutung Information über Vor- und Nachzustand. Auch das Töten von (unbestimmt vielen) Geiseln endet für jede einzelne Geisel mit dem Tod. Ein Töten-Ereignis wird in einer nichtbegrenzten Anzahl wiederholt und damit sekundär zum Elementarereignis in einem Prozeß.

(30c): Beide Sätze denotieren ein Ereignis, erst durch die explizite Zeitspannenangabe wird eine iterative Lesart erzwungen.

Gegen Auffassung A möchte ich die zwei Arten von Iteration bei der Bedeutungskonstitution in Steinitz (1981) ins Feld führen, die ich immer noch für gültig halte. 
1. Ein Zustand oder ein Prozeß kann einfach oder komplex sein im Sinne des inneren Zeitbezugs: Schlafen, arbeiten u.ä. denotieren einfache, nicht zerlegbare Situationen (analog zu Massenomina). Atmen, flattern, husten, schreiten denotieren komplexe Situationen, die intern als kontinuierliche Abfolge gleichartiger Elementarvorgänge ("einen Atemzug / Flügelschlag usw. tun") analysierbar sind. Diese Situationen weisen eine Körnung auf, hier ist Iterativität konstitutiv für die Verbbedeutung, hier spreche ich von innerer Iterativität. Für die situative Einordnung ist aber diese Körnung nicht relevant, flattern, fliegen, schreiten und fahren gehören mit modifikatorischen Nuancen derselben Subklasse Bewegungsverb an, sie denotieren alle die "continuity of events of motion" (Jackendoff (ibid.), in 7.2. zitiert), egal ob die Bewegung eine Körnung aufweist oder nicht, wie etwa in Der Airbus flog in großer Höhe. Von dieser Art scheint mir auch Peter wurde größer zu sein. Jede Zeitspanne, in der Peters Größe gegenüber einer davorliegenden zugenommen hat, ist willkürlich herausgegriffen und läßt sich weiter teilen. Nicht mögliche Elementarvorgänge, sondern nur der durch das Verb bzw. die Kopula-Konstruktion denotierte Gesamtvorgang referiert (zusammen mit seinen Komplementen) auf eine Situation, und zwar auf eine atelische.

Die Körnung einer atelischen Situation wird erst relevant, wenn ein Elementarvorgang herausgegriffen werden soll. In Aktionsartensprachen tut der Semelfaktiv genau dies, vgl. $d y$ šat' : $d y c h n u t^{\prime}$ "einen Atemzug tun", kopnut' "einen Spatenstich tun".

2. Eine Gesamtsituation kann komplex sein im Sinne des äußeren Zeitbezugs: Hierher gehören die Beispiele in (30c) und oft schlafen, immer wieder auf den Ast flattern, es können also sowohl telische wie atelische Verben betroffen sein. Es werden Einzelsituationen quantifiziert. Die Quantifikation erfolgt relativ $\mathrm{zu}$ einem vom inneren Zeitbezug unabhängigen Zeitraum, der extern determiniert ist. Die Abfolge der Einzelsituationen verläuft diskontinuierlich. Iterativität ist hier nicht konstitutiv für die Verbbedeutung, sondern modifiziert den ganzen Ausdruck temporal, hier spreche ich von äußerer Iterativität. Die mögliche Verschiebung eines spezifischen Situationstyps durch äußere Iterativität ist ein Fall von sekundärer Situationstyp-Modifikation. Äußere Iteration ist für alle Verbklassen möglich. Das folgende Beispiel illustriert den Unterschied, der nicht verwischt werden sollte:

\section{(Petra bemühte sich abzunehmen,)}

a) aber statt dessen nahm sie mehr und mehr/wochenlang zu

b) aber sie nahm immer/mehrmals wieder zu

innere Iterativität äußere Iterativität

Bei (31a) muß Petras Gewicht zu einem beliebig gewählten Einschnitt höher sein als zu einem Zeitpunkt davor. Bei (31b) könnte Petra dagegen immer wieder auf das Anfangsgewicht zurückkommen.

Äußere Iterativität ist ein Fall für Aktionsarten in Sprachen, die eine solche grammatische Kategorie haben, vgl. Russ. videt' (Perfektiv) : vidyvat' (Imperfektiv, iterative Aktionsart) "oft sehen, zu sehen pflegen", My razgovarivali "Wir sprachen oft mit einander". Aktionsartensprachen bilden von einem aktionsartneutralen Basisverb Iterative nur im Sinne der äußeren Iterativität, für innere Iterativität wie atmen wird auf keinen Fall ein Iterativ-Affix genommen. Das Deutsche verfügt für diese Art Iterativität über lexikalische Umschreibungen die den Situationstyp relativieren. Näheres dazu in Isačenko (1962) und Steinitz (1981).

\subsubsection{Alternation von Prozeß und Ereignis}

Werden-Konstruktionen mit relativen Adjektiven haben mit anderen Verbklassen (Bewegungs- und SUK-Verben) die Eigenschaft gemeinsam, zwischen Prozeß- und EreignisPrädikat alternieren zu können. Bei diesen Verbklassen kommt allerdings keiner kommentar- 
los auf die Idee, die Prozeß- aus der Ereignis-Variante abzuleiten, wie es nach Auffassung A bei werden geschieht.

\section{Vergleichsstück SUK-Verben}

DP-formatige Komplemente haben Einfluß auf den Situationstyp eines Ausdrucks, der Prozesse denotiert, in denen ein Objekt nach und nach dem Ereignis unterzogen wird (inkrementelle Objekte). Objektteile entsprechen Teilen des Ereignisses. Trinken und sein Komplement stehen in solch einer Relation des Sukzessiv-Patiens (SUK-Verben), vgl. Krifka (1989). Krifka basiert seine Analyse von Zeitkonstitution auf der bekannten Parallele von atelischen, in seiner Terminologie kumulativen Verben und kumulativen Massennomen/pluralischen Individualnomen einerseits und der von telischen Verben und gequantelten (zählbaren) Nomen andererseits. Die Verben essen, trinken, malen sind nach Krifka (1989:155ff.) kumulativ, also isoliert atelisch, Prozeßverben. Die Sätze

$$
\begin{aligned}
& \text { Er trinkt Wein } \\
& \text { Er ißt Äpfel / Obst }
\end{aligned}
$$

sind kumulativ, weil die DP-formatigen Komplemente kumulative Plural-Prädikate ( $\ddot{\text { Apfel }}$ ) oder kumulative Singular-Prädikate (Wein, Obst) sind.

\section{Er trinkt ein Glas Wein ißt einen Apfel}

sind dagegen telisch, weil die DP-formatigen Komplemente gequantelt sind. ${ }^{10}$ Die Gequanteltheit bzw. Kumulativität der Komplement-DPs transitiver Verben bestimmen den Gesamtausdruck als Accomplishment bzw. Prozeß. Ähnlich verfährt Tenny (1994).

Werden alterniert in gleicher Weise je nach Art des Komplements zwischen beiden Situationstypen. Es gibt aber einige Unterschiede: werden kommt (abgesehen von Wendungen wie Das wird schon) nicht komplementlos vor und ist deshalb bezüglich der Kumulativität (in anderer Terminologie Prozeßhaftigkeit) unterspezifiziert. Die Bedeutung komparativer Adjektive, die eine Vergleichsinstanz inhärieren, bewirkt, daß größer werden ambig ist und mit einer rekonstruierbaren Vergleichsinstanz telisch ist (vgl. Abschnitt 6.1.1).

\section{Vergleichsstück Bewegungsverben}

Wie die Kopula- haben Bewegungsverben in ihrer Semantischen Form ein durch die Variable $P$ repräsentiertes Prädikat-Argument, das durch Präpositionalphrasen realisiert werden kann. Auch Bewegungsverben changieren je nach Art des PP-Komplements zwischen Prozeß und Accomplishment. Bewegt sich ein Objekt fort, dann erzeugt es einen Weg (vgl. auch Kaufmann (1995)), egal ob es im Kreise geht, ziellos herum schweift, eine bestimmte Richtung einschlägt oder sogar ein Ziel anpeilt. Der Weg gehört zur Bedeutungskonstitution des Bewegungsverbs selbst, unabhängig von seinem Komplement. ${ }^{11}$ Im Normalfall wird der Weg selbst sprachlich nicht spezifiziert, im Spezialfall, wie in Er fuhr viele Umwege, Er hatte einen weiten Weg zurückgelegt, kann der Weg als sog. Innerer Akkusativ ( wie in den Schlaf des Gerechten schlafen) explizit gemacht werden.

\footnotetext{
${ }^{10}$ Krifka weist darauf hin, daß ein solcher Einfluß des Komplement-Typs nur für SUK-Verben gilt. Bei sehen und anderen atelischen Verben beeinflußt das Objekt in keiner Weise die Atelizität des Verbs, vgl. Er sah das Schiff noch stundenlang / *in einer Stunde; der Ausdruck verhält sich wie ein Zustandsprädikat, vgl. Abschnitt 4.

${ }^{11}$ Alle mir bekannten Arbeiten zu diesem Thema gehen dagegen davon aus, daß Wege durch Orte definiert sind, die durch direktionale PPs zu spezifizieren sind. In Bierwisch (1988:25ff) sind Wege mittels Intervallschachtelung strukturierte Lokationen, wobei „Init“ (aus dem Haus) den Anfang des Weges und „Fin“ (in die Stadt) die längste Teilstrecke repräsentiert.
} 
Die Fortbewegung eines Objektes und damit der erzeugte Weg bekommt explizit eine Richtung durch Ausdrücke wie vorwärts, nach Süden als mögliche Spezifikation von P. Für das Gesamtprädikat gilt, was für homogene Entitäten gilt: "südwärts laufen" ist zum Zeitintervall $\mathrm{T}$ wahr, wenn es zu allen Teilintervallen von T wahr ist (Dowty (1979)), der Ausdruck ist atelisch. Eine Begrenzung der Bewegung kommt erst zustande durch Nennung des Ziels, wo die Fortbewegung und damit der Weg endet. Dies leisten die sog. Richtungsangaben, wie in die Stadt, die angemessener Zielangaben zu nennen wären. In die Stadt laufen ist telisch.

Die Parallelitäten zwischen SUK-Verben, Bewegungsverben und werden bezüglich der Alternation von Prozeß und Accomplishment liegen auf der Hand. Für alle drei Klassen gilt:

- Sie sind bezüglich Telizität unterbestimmt.

- Der Komplementtyp entscheidet, ob der Gesamtausdruck atelisch oder telisch ist, ob er also vom Situationstyp Prozeß oder Ereignis ist.

Die Ausdrücke (stadteinwärts) laufen und größer/dunkler werden (wie gesagt: ohne rekonstruierbare Vergleichsinstanz) verhalten sich wie Obst/Äpfel essen; die Ausdrücke in die Stadt laufen und groß/dunkel werden verhalten sich wie einen Apfel essen, das bestätigen der bekannte Test mit den zwei Arten Zeitdauer- und Zeitspannen-Adverbiale und der Koordinationstest:

DP: $\quad$ a) Peter aß stundenlang Äpfel

* Er aß in einer Minute Äpfel

b)*Er aß stundenlang drei Äpfel

Er aß in einer Minute drei Äpfel

PP: $\quad$ a) Er lief stundenlang (in) Richtung Norden

*Er lief in zwei Stunden in Richtung Norden

b) *Er lief stundenlang in die Stadt

Er lief in zwei Stunden in die Stadt

AP: a) Es wurde zehn Minuten (lang) dunkler

${ }^{*}$ Es wurde in zehn Minuten dunkler

b) *Es wurde zehn Minuten (lang) dunkel ${ }^{12}$ Es wurde in zehn Minuten dunkel
a) Er aß und aß (̈̈pfel)
Er lief und lief (vorwärts)
Sie wurde größer und größer ${ }^{13}$
Er wuchs und wuchs
b) *Er aß und aß drei Äpfel
${ }^{*}$ Er lief und lief in die Stadt
* Sie wurde gro $\beta$ und gro $\beta$
*Er alterte und alterte

In Sie wurde größer und größer bzw. Sie wurde immer größer ist die Möglichkeit, daß der Kontext einen bestimmten Vergleichswert liefert, sprachlich ausgeschlossen, es gibt nur eine

\footnotetext{
${ }^{12}$ Wem die beiden besternten Sätze in Ordnung zu sein scheinen, der muß berücksichtigen, daß das Adverbial in zehn Minuten in a) anders als in b) die Zeitspanne angibt, innerhalb der das Dunklerwerden einsetzt, präziser in innerhalb von zehn Minuten ausgedrückt. In Es wurde zehn Minuten (lang) dunkel sind wiederum die Skopusverhältnisse verändert. Nicht das Ereignis selbst, sondern der Nachzustand steht im Skopus von zehn Minuten, das macht die synononyme explizitere Form Es wurde für zehn Minuten dunkel deutlich. Bei Achievementverben ist diese Form die allein mögliche, vgl. Er betrat *(für) zehn Minuten das Zimmer.

${ }^{13}$ Das gleiche Ergebnis bringen Intensivierer wie immer in Er wurde immer größer vs. *Er wurde immer groß, vgl. Lindström (1997).
} 
atelische Lesart. Ansonsten ist werden + komparative AP aufgrund der Variabilität des Vergleichswertes in der Bedeutung des Adjektivs ambig:

- ein kontextuell vorgegebener Vergleichswert bewirkt Telizität der Konstruktion,

- ohne einen solchen wird durch innere Iteration Atelizität bewirkt.

Es ist aber ein Unterschied zwischen DPs und Ziel-PPs bzw. APs im Positiv zu beachten:

- Aufgrund ihres Prädikat-Charakters benennen nur PPs wie in die Stadt und APs wie groß den Nachzustand, auf den der Prozeß abzielt, "in der Stadt sein" bzw. "groß sein".

- Gequantelte DPs wie drei Äpfel bestimmen dagegen die Begrenztheit eines Prozesses "essen" dadurch, daß "das Objektdenotat dem Verbereignis nach und nach unterzogen wird", bis es in diesem Falle verschwunden ist. Der Ausdruck drei Äpfel essen sagt aber nichts über einen Nachzustand, der muß extra benannt werden wie in (36a).

Darin gleichen die DP-Argumente den allerdings modifikatorischen Gradangaben oder Zeitspannenadverbien bei allen atelischen Verben, vgl. (36b)

(36) a) Peter aß drei Äpfel / Er aß eine Stunde lang und hörte dann auf/ und war dann satt / und ging dann joggen / ...

b) Er wuchs drei cm / lief zwei km und blieb dann stehen

Bei werden und Bewegungsverben ist dagegen im Komplement (Ziel-PP bzw. AP im Positiv) ein spezifischer Nachzustand genannt, es muß also auch ein Zustandswechsel involviert sein.

\section{Spezifikation von werden-Konstruktionen}

\subsection{Zusammenfassung:}

AP +sein

- Sein-Konstruktionen sind Zustandsprädikate, unabhängig von der Subklasse ihres PrädikatArguments, sie denotieren Situationen als homogen und statisch.

- Wenn eine homogene Situation zum Zeitintervall T wahr ist, dann ist sie auch zu allen Teilintervallen von T wahr. Homogene Prädikate enthalten keine Relation zu Propositionen außerhalb der Situationszeit T, d.h. zu solchen, die auf Nachbarsituationen (Vor- und Nachzustand) referieren, und damit keinerlei Festlegung über Anfang oder Ende der beschriebenen Situation.

\section{$\mathbf{A P}+$ werden}

- Werden beschreibt unabhängig von der Art seiner Komplemente eine Situation als nichtstatisch, als Veränderung, in der es keine Teilsituationen mit gleichen Werten der im Prädikat-Argument genannten Eiegnschaft gibt. Werden ist von der unmarkierten statischen Kopula sein unterschieden durch das Prädikat CHANGE.

- CHANGE ist unterbestimmt bezüglich Telizität und Prozeßhaftigkeit. Die Spezifizierung kommt durch das Komplement zustande.

- Eine AP im Komparativ (Subklasse $\mathrm{P}_{\text {relk }}$ ) bezeichnet eine Eigenschaft mit variablem Wert, zusammen mit werden ist der Veränderung eine Richtung, aber ohne rekonstruierbaren Vergleichswert keine Begrenzung gegeben, und damit ist kein Nachzustand in Sicht. Eine Veränderung ohne Begrenzung ist ein Prozeß.

- Eine AP im Positiv (die Subklassen $\mathrm{P}_{\text {relp }}$ und $\mathrm{P}_{\text {abs }}$ ) bezeichnet einen festen Wert einer Eigenschaft. Zusammen mit werden wird dieser Wert als Ziel- oder Nachzustand 
interpretiert. Eine Veränderung mit einem implizierten Nachzustand ist telisch, ein Ereignis.

- Werden im Kontext eines absoluten Adjektivs ist markiert durch den unmittelbaren Übergang von Nicht-P zu P, der Wechsel läßt keine Prozeßhaftigkeit zu, er ist atomar.

- BECOME als telisches Prädikat verstanden ist im Verb nicht enthalten, geschweige denn im Komplement.

Damit haben wir die Ingredienzien für die Spezifizierungen der behandelten KopulaKonstruktionen beisammen. Ich bin mir aber bewußt, daß ich mich immer noch im Vorfeld einer befriedigenden formalen Darstellung bewege.

\subsection{Bedeutungsanreicherung}

\section{Telische/inhomogene Veränderung}

„groß werden“, „schwanger werden" sind inhomogene Prädikate, die Prädikat-Argumente von werden haben als lexikalischen Kopf ein Adjektiv im Positiv (relatives $\left(\mathrm{P}_{\text {relp }}\right)$ bzw. ein absolutes Adjektiv $\left(\mathrm{P}_{\mathrm{abs}}\right)$ ). Deren inhärente Eigenschaft, einen festen Abschnitt auf einer Skala $\mathrm{zu}$ bezeichnen, bekommt im Kontext von werden die Funktion, das Ziel (TARGet) der Veränderung zu denotieren. Für die jeweilige Semantische Form eines relativenAdjektivs im Positiv oder eines absoluten Adjektivs stehen die Abkürzungen $\mathrm{P}_{\text {relp }}$ bzw. $\mathrm{P}_{\mathrm{abs}}$. CHANGE Px wird in diesem Kontext durch das Prädikat TARG angereichert. Es gilt das Bedeutungspostulat:

$$
\forall \mathrm{s} \exists \mathrm{s}^{\prime}\left[\mathrm{s}^{\prime} \text { TARG } \mathrm{s}\right] \leftrightarrow \exists \mathrm{P}_{\text {relp/abs }} \exists \mathrm{x} \text { [s INST [CHANGE Px]] }
$$

Damit ist über das tatsächliche Erreichen des Zielzustandes nichts festgelegt, diese Einschränkung erweist sich in Aspektsprachen als relevant. Die Komponente BECOME von Auffassung A erlaubt keine derartige Differenzierung zwischen erreichtem (perfektiv) und nicht erreichtem (imperfektiv) Zielzustand.

\section{Atomare Veränderung}

„schwanger werden“" ist ein Prädikat atomarer Veränderung. Aus der Eigenschaft absoluter Adjektive $\left(\mathrm{P}_{\mathrm{abs}}\right)$, in kontradiktorischer Beziehung zu ihrem Antonym zu stehen, ist abzuleiten, daß die Veränderungssituation s nicht ausdehnbar, sondern punktuell (ATOMar) ist, d.h. es gibt keine Teilsituation $s_{\mathfrak{i}}$ von $s$. Unter dieser Bedingung wird die Prädikation um eine weitere Komponente ATOM angereichert:

$$
\forall \mathrm{s}[\mathrm{ATOM} \mathrm{s}] \leftrightarrow \exists \mathrm{P}_{\mathrm{abs}} \exists \mathrm{x}\left[\mathrm{s} \text { INST }[\text { CHANGE Px }] \& \neg \exists \mathrm{s}_{\mathrm{i}}\left[\mathrm{s}_{\mathrm{i}} \subset \mathrm{s}\right]\right]
$$

\subsection{Situationstypspezifizierung}

Die Situationstypen sind für mich Namen für bestimmte Konstellationen, die sich aus der Komposition der Wortbedeutungen zusammen mit den Bedeutungspostulaten ergeben. Es gelten folgende Definitionen:

$$
\forall \mathrm{s}\left(( \text { Zustand } \mathrm { s } ) \leftrightarrow \mathrm { df } \forall \mathrm { P } \forall \mathrm { x } \forall \mathrm { s } _ { \mathrm { i } } \left[[ \mathrm { s } \text { INST Px } ] \& \mathrm { s } _ { \mathrm { i } } \subset \mathrm { s } \rightarrow \left[\mathrm{s}_{\mathrm{i}}\right.\right.\right. \text { INST Px]]) }
$$

Veränderungssituationen sind Nicht-Zustände, markiert durch das Prädikat CHANGE.

Veränderungssituationen mit einem Target sind Ereignisse.

(40) $\quad \forall \mathrm{s}\left(\left(\right.\right.$ Ereignis s) $\leftrightarrow$ df $\exists \mathrm{P} \exists \mathrm{x}\left[[\mathrm{s}\right.$ INST CHANGE Px $] \& \exists \mathrm{s}^{\prime}$ [s' TARG s]]) 
Prozesse haben kein Target, sie sind durch CHANGE hinreichend charakterisiert.

Achievements sind atomare Ereignisse.

(41) $\forall \mathrm{s}\left(\left(\right.\right.$ Achievement s) $\leftrightarrow$ df $\exists \mathrm{s}^{\prime}\left[\mathrm{s}^{\prime}\right.$ TARG s] \& [ATOM s])

Accomplishments sind durch TARG hinreichend charakterisiert.

Die zunehmend einschränkenden Bedingungen, repräsentiert durch die Prädikate CHANGE, TARG und ATOM, ergeben folgende Hierarchie der Situationstypen:

( $\mathrm{LE}=$ Lexikoneintrag, $\mathrm{BP}=$ Bedeutungspostulat) 
(42)

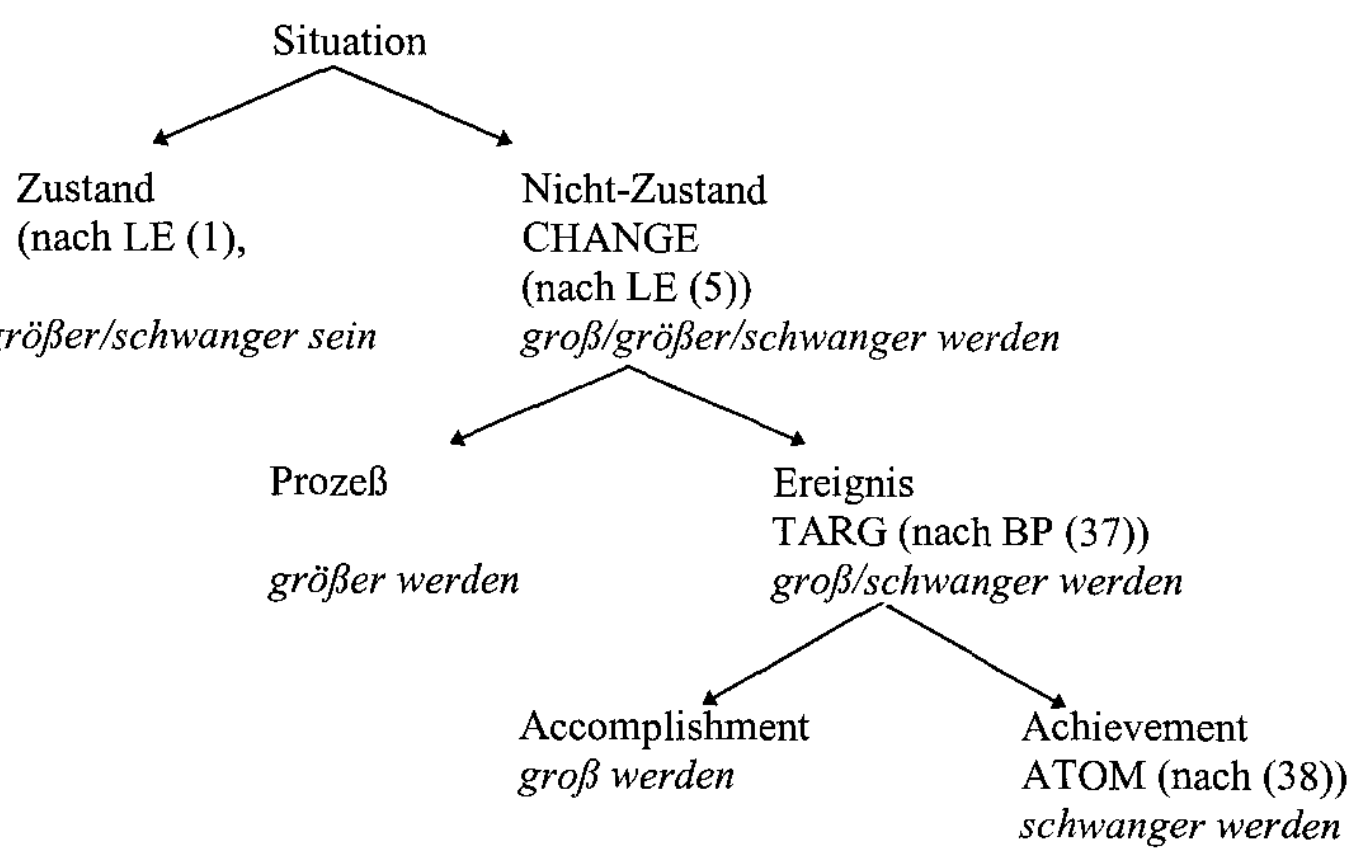

Unter der Hand bekommen wir die Beschreibung von Markiertheitsverhältnissen zwischen den Situationstypen, wie sie mit nur zwei semantischen Klassifizierungskomponenten der Art von Tabelle (33) in Abschnitt 4.1 ( \pm Prozeß und \pm Become) nicht darstellbar wären. Mit Zunahme der semantisch einschränkenden Prädikate (in anderer Notation: der positiv markierten Merkmale), die einen Situationstyp identifizieren, nimmt auch die Markiertheit des Typ zu:

(43)

\begin{tabular}{|l|c|c|c|}
\hline Situationstyp & CHANGE Px & s'TARG s & ATOM s \\
\hline Zustand & & & \\
\hline Prozeß & + & & \\
\hline Accomplishment & + & + & \\
\hline Achievement & + & + & + \\
\hline
\end{tabular}

[Manuskriptabgabe 10.03.1999] 


\section{Literaturverzeichnis}

Amrhein, Jürgen (1999): Zur Semantik von werden. Vortrag im SFB-Workshop „Kopulaverben und Prädikative. Bierwisch, Manfred (1987): Semantik der Graduierung. In: M.Bierwisch/E.Lang (eds.) 91-286.

- (1988): On the Grammar of Local Prepositions. In:M.Bierwisch/W.Motsch /I.Zimmermann (eds.), 1-65 .

- / E.Lang (eds.) (1987): Grammatische und konzeptuelle Aspekte von Dimensionsadjektiven (= studia grammatica XXVI/XXVII). Berlin: Akademie-Verlag.

- /W.Motsch/ I.Zimmermann (eds.) (1988): Syntax, Semantik und Lexikon (-studia grammatica XXIX) Berlin: Akademie-Verlag.

Bäuerle, Rainer (1994): Zustand-Prozeß-Ereignis. Zur Kategorisierung von Verb(alphras)en. Wuppertaler Arbeiten zur Sprachwissenschaft 10,1-32

Comrie, Bernhard (1976): Aspect. Cambridge: Cambridge University Press.

Demjjanov, Assinja (1998): Eine semantische Untersuchung der Perfektivierungspräfigierung. Diss. HumboldtUniversität Berlin.

Dölling, Johannes (1997): Ist die Kopula mehrdeutig? Anmerkungen zu einem Vorurteil. In: U.Scheffler und K. Wuttich (eds.):Terminigebrauch und Folgebeziehung, Berlin: Logos, 5-24.

Dowty, David (1979): Word meaning and Montague Grammar. The Semantics of Verbs and Times in Generative Semantics and in Montague's PTQ. Synthese Language Library, Vol.7. Dordrecht: Reidel Publ. Company.

Heim, Irene (1985): Notes on comparatives and related matters. Ms., University of Texas.

Herweg, Michael (1991a): Perfective and imperfective aspect and the theory of events and states. Linginuistics. 29: 969-1010.

- (1991b): Temporale Konjunktionen und Aspekt. Kognitionswissenschaft 2:51-90.

Isačenko, Alexander (1962): Die russische Sprache der Gegenwart. Teil I Formenlehre. Halle: Niemeyer.

Jackendoff, Ray (1993): Semantics and Cognition. Current Studies in Linguistics Series, Vol. 8. Cambridge (Mass), London: The MIT Press, 6. Aufl.

- (1996): The Proper Treatment of Measuring Out, Telicity, and Perhaps Even Quantification in English. Natural Language \& Linguistic Theory 14/2, 305-354.

Jäger, Gerhard (in Vorbereitung): Eine alternative Analyse von werden.

Kamp, Hans und Uwe Reylc (1993): From Discourse to Logic, Part 2. Kluwer Academic Publishers.

Kaufmann, Ingrid (1995): Konzeptuelle Grundlagen semantischer Dekompositionsstrukturen: Die Kombinatorik lokaler Verben und prädikativer Komplemente. Tübingen: Niemeyer (= Linguistische Arbeiten 335), 258pp.

Klein, Ewan (1980): A semantics of positive and comparative adjectives. Linguistics and philosophy 4:1-45.

- (1991): Comparatives. In: A.v. Stechow/D.Wunderlich (ed): Semantik: ein internationales Handbuch der zeitgenössischen Forschung, 673-691.

Klein, Wolfgang (1992): Tempus, Aspekt und Zeitadverbien. Kognitionswissenschaft Band2 Heft 3/4, $107-118$.

- (1994): Time in Language. London: Routledge.

Koch/Rosengren (1995) Secondary Predications: Their Grammatical abd Conceptual Structure. Sprache und Pragmatik 35, 100p.

Kratzer, Angelika (1994): The Event Argument and the Semantics of Voice. University of Massachusetts, Amherst (Manuskript)

Krifka, Manfred (1989): Nominalreferenz und Zeitkonstitution. Zur Semantik von Massentermen, Pluraltermen und Aspektklassen. München: Fink Verlag, 261p.

- (1989a): Nominalreferenz, Zeitkonstitution, Aspekt und Aktionsart: Eine semantische Erklärung ihrer Interaktion. In: W.Abraham, Th.Janssen (eds.): Tempus - Aspekt - Modus. Tübingen: Niemeyer, 227-258.

Lang, Ewald (1993): Duale Operatoren. Teilprojekt D2 des Sonderforschungsbereichs 282, Theorie des Lexikons, Bergische Universität Gesamthochschule Wuppertal, Finanzierungsantrag 1994-96.

Lang, Ewald \& Cristina Schmitt \& Renate Steinitz (in Vorbereitung): REMAIN and BECOME

Levin, B. \& T.R. Rappaport Hovav (1991): The Lexical Semantics of Verbs of Motion. In: I. Roca (ed.): Thematic Structure: Its Role in Grammar. Berlin: de Gruyter.

Leys, Odo (1989): Aspekt und Rektion räumlicher Präpositionen. Deutsche Sprache 2: 97-113.

Lindström, Jan K. (1997): Intensification of the comparative. In Linguistlist.org, 04.07.97.

Löbner, Sebastian (1989): German schon-erst-noch: An integrated analysis. Linguistics and Philosophy, 12:167212.

Maienborn, Claudia (1994): Kompakte Strukturen: Direktionale PPn und nicht-lokale Verben. In: Felix, S/ Ch. Habel / G Rickheit (eds.): Kognitive Linguistik. Repräsentation und Prozesse. Opladen: Westdeutscher Verlag, 229-249.

- (1996): „Für Renate“ Vortrag auf einem Kolloquium des FAS im März 1996.

Marr, David (1982): Vision, San Francisco: Freeman.

Musan, Renate (1999): Zur Semantik von werden. Ist prädikatives werden transitional? Ms. HumboldtUniversität Berlin. 
Piñon, Christopher (1996): Reicher werden and reich werden. Workshop Prädikativkonstruktionen am FAS Berlin.

- (1997): Achievements in an Event Semantics. First extended version of SALT VII paper, 9 July 1997.

- (1997a): Allmählich. Vortrag auf der Tagung Sinn und Bedeutung 1997.

Pustejovsky, James (1991): The syntax of event structure. Cognition 41, 47-81.

Schlachter, Wolfgang (1968): Arbeiten zur strukturbezogenen Grammatik. München: Fink Verlag.

Steinitz, Renate (1975): Sind alle Inchoativa inchoativ? In: Linguistische Studien des ZISW der Akademie der Wissenschaften der DDR, Heft 18: 1-82.

- (1981): Der Status der Kategorie "Aktionsart" in der Grammatik (oder: Gibt es Aktionsarten im Deutschen?) (= Linguistische Studien des ZISW der Akademie der Wissenschaften der DDR, Reihe A, H. 76): 122 pp. (1981).

- (1985): Zur Struktur und Funktion des Lexikons in der Grammatik. In: Linguistische Studien des ZISW der Akademie der Wissenschaften der DDR, Heft 127: 1-42.

- (1989): $\mathrm{V}^{\mathrm{u}}, \mathrm{I}^{\mathrm{y}}$ und $\mathrm{I}^{\mathrm{Z}}$ : Überlegungen zum Prädikativ. In: W. Motsch (ed): Linguistische Studien des Zentralinstituts für Sprachwissenschaft der AdW der DDR, Reihe A, H. 194. Berlin, 210-234.

- (1990): Prädikation, Modifikation und die Adverbiale. In Linguistische Studien des ZISW der Akademie der Wissenschaften der DDR, Heft 206: 117-132.

- (1992): Durative und inchoative Prädikate und die Adverbialkomplemente von Verben. In: Hoffmann, Ludger (eds.): Deutsche Syntax. Ansichten und Aussichten, Berlin, New York: de Gruyter, 186-205.

- (1997a): Lexikalische Kategorisierung: Ein Vorschlag zur Revision. In: Löbel, Elisabeth / Gisa Rauh (eds.): Lexikalische Kategorien und Merkmale. Lingistische Arbeiten 366:1-26.

- (1997b): Valenznotwendige Präpositionalphrasen: weder Argument- noch Adjunktposition. In: Dürscheid, Christa / Karl-Heinz Ramers / Monika Schwarz (eds.): Sprache im Fokus. Festschrift füe Heinz Vater. Tübingen: Niemeyer 329-350.

Steube, Anita (1995): Formale Verfahren der linguistischen Modalitätsbeschreibung. In: Jachnow, Helmut/ M. Wingender (eds.): Temporaltät und Tempus. Wiesbaden: Harrassowitz Verlag, 70-111.

- (1997): Der russische Aspekt und die Ereignisrolle des Verbs. In: U. Junghanns, / G. Zybatow, (eds.): Formale Slavistik. Leipziger Schriften zur Kultur-, Literatur-, Sprach- und Übersetzungswissenschaft. Frankfurt/M.: Vervuert Verlag, 213-227.

Talmy, Leonard (1985):Lexicalization Patterns: Semantic Structure in Lexical Forms. In: Shopen, Timothy (ed.): Language typology and syntactic description. Volume III: Grammatical categories and the lexicon.. Cambridge, London, New York, New Rochelle, Melborne, Sydney: Cambridge Univesity Press, 57-149.

Tenny, Carol L. (1994): Aspectual Roles and the Syntax-Semantics Interface. Studies in Linguistics and Philosophy 52. Dordrecht / Boston / London: Kluwer Academic Publishers, 245 p.

Vendler, Zeno (1967): Verbs and Times. Linguistics in Philosophy. Ithaca: Cornell University Press.

Verkuyl, Henk J. (1995): Apectualizers and Event Structure. UIL OTS Working Papers November 1995.

- (1997): Events as Dividuals: Aspectual Composition and Event Semantics. UIL OTS Working Papers January 97.

von Stechow, Arnim(1983): Sind 'groß' und 'klein' Prädikate oder Relationen? Ein Interview mit Aristoteles. In: Allgemeine Sprachwissenschaft, Sprachtypologie und Textlinguistik. Festschrift für Peter Hartmann. Tübingen: Narr, 105-120.

- (1984): Comparing Semantic Theories of Comparison. Journal of Semantics 3, 1/2: 1-77.

- (1996): The Different Readings of Wieder 'Again': A Structural Account, Journal of Semantics 13,2: 87-138.

Wunderlich, Dieter (1994): Models of Lexical Decomposition. In: Edda Weigand \& Franz Hundsnurscher (eds.) Lexical Structures and Language Use, 169-183. Tübingen: Niemeyer 1996.

- (1997): CAUSE and the Structure of Verbs. Linguistic Inquiry 28/1, 27-68.

- Michael Herweg (1991): Lokale und Direktionale. In Armin von Stechow und Dieter Wunderlich (eds.) Semantik. Ein internationales Handbuch der zeitgenössischen Forschung. Berlin: de Gruyter.

Zimmermann, Ilse (1987): Zur Syntax von Komparativkonstruktionen. In: Bierwisch, M./Lang, E (eds.), 29-90.

- (1988): Die substantivische Verwendung von Adjektiven und Partizipien. In: M.Bierwisch/ W.Motsch./ I.Zimmermann (eds.), 279-311.

- (1992): Der Skopus von Modifikatoren. In: I.Zimmermann und A.Strigin (eds.): Fügungspotenzen (=studia grammatica XXXIV), 251-27.

- (1998a): Das deutsche Partizip II, sein Verhältnis zum Aspekt, zum Passiv und zu den Adjektiven. Vortrag 5.6.1998 am ZAS Berlin.

- (1998b): Die Integration topikalischer DPs in die syntaktische und semantische Struktur von Sätzen. Ms. 